\title{
ChemComm
}

Cite this: Chem. Commun., 2013, 49, 2594

Received 3rd December 2012, Accepted 5th February 2013 DOI: $10.1039 / \mathrm{c3cc38671f}$

www.rsc.org/chemcomm

\section{The making of iLiquids - the chemist's equivalent of the iPhone}

\author{
Zhaofu Fei and Paul J. Dyson
}

\begin{abstract}
In this viewpoint we discuss the contributions from John S. Wilkes and Michael J. Zaworotko concerning the discovery of stable ionic liquids (ILs) and their accompanying structural studies (J. Chem. Soc., Chem. Commun., 1992, 965) and Robin D. Rogers and James H. Davis et al. who described the rational modification of ILs for specific applications - so-called task-specific ionic liquids (TSILs) (Chem. Commun., 2001, 135). These were quite simply groundbreaking discoveries that inspired the scientific community, leading to enormous growth in the field and the wide and diverse range of ILs and their applications. The multitude of 'apps' of ILs warrants an analogy with the iPhone and our use of the term iLiquids.
\end{abstract}

Many reviews on ILs commence with a 'historical' introduction and in our opinion one of the finest accounts is provided by John S. Wilkes. ${ }^{1}$ The story begins, at least in a semi-rational way, with ethylammonium nitrate (mp: $12-14{ }^{\circ} \mathrm{C}$ ) almost 100 years ago, ${ }^{2}$ with sporadic reports on the use of ILs as media for electrochemical studies and, less commonly, as solvents for organic reactions, over the next half century or so. The ionic liquids used in these studies were based on chloroaluminates, identified in Friedel-Craft reactions:' ${ }^{3}$ 'It is proposed that the high solubility of aluminum halides in these Friedel-Crafts complexes is due to the formation of a series of higher complexes of this kind, with the general formula $R^{+}\left[A l X_{4} \cdot n A l X_{3}\right]^{-}$. It is further proposed that these complexes play an important role in most Friedel-Crafts reactions by furnishing a highly polar medium in which the ionic intermediates may form and react.' The United States Air Force Academy pioneered extensive research on ILs in the 1960's and 70's, largely related to batteries, with the discovery of new classes of chloroaluminates. ${ }^{4}$

In the late 1970's Jerry Atwood's group at the University of Alabama began to systematically study the alkylaluminates - they coined the term liquid clathrates to describe them. ${ }^{5}$ Michael $\mathrm{J}$. Zaworotko and Robin D. Rogers both graduated from Atwood's group in 1982 and it was in this same year that John S. Wilkes published a seminal paper describing a series of (low melting) dialkylimidazolium chloroaluminate salts. ${ }^{6}$

Despite more than half a century of effort, research on ILs was conducted by a relatively small number of groups - at that time mostly based in the US. This lack of interest from a broader research community may be attributed to the poor stability of the ILs known at that time (mostly chloroaluminates) and their

Institut des Sciences et Ingénierie Chimiques, Ecole Polytechnique Fédérale de Lausanne (EPFL), CH-1015 Lausanne, Switzerland. E-mail: paul.dyson@epfl.ch limited envisaged applications. The two communications that we highlight in this viewpoint explicitly addressed both of these limitations: Wilkes and Zaworotko showed how stable ILs could be prepared and Rogers and Davis et al. demonstrated that ILs could be rationally modified for specific applications. With hindsight we can conclude that it was obvious that more stable ILs were required and that stable ILs would increase their range of applications, but at that time these ideas were revolutionary. In addition, a greater understanding of the structure of the ILs would also allow ILs to be prepared in a rational manner ultimately leading to designer solvents. Arguably, these papers did more than any other to generate today's considerable interest in ILs in a vast range of fundamental and applied settings.

The article by Wilkes and Zaworotko published in 1992 described air and water stable 1-ethyl-3-methylimidazolium, [Emim $]^{+}$, ILs composed of $\mathrm{NO}_{3}{ }^{-}, \mathrm{NO}_{2}{ }^{-}, \mathrm{BF}_{4}{ }^{-}, \mathrm{MeCO}_{2}{ }^{-}$and $\mathrm{SO}_{4}{ }^{2-}$ anions in place of chloroaluminate anions. ${ }^{7}$ The idea was simple, almost obvious, and yet nobody else thought to do it. Moreover, the synthetic approach was also very simple (so simple that a schematic of the route was not even shown), with the desired salts being prepared in essentially quantitative yield via metathesis of 1-ethyl-3-methylimidazolium iodide ([Emim]I) and the appropriate silver salt (Scheme 1), followed by simple removal of the AgI precipitate. The route used readily available starting materials and compared to the chloroaluminates very little purification was required. Not all the ILs described were

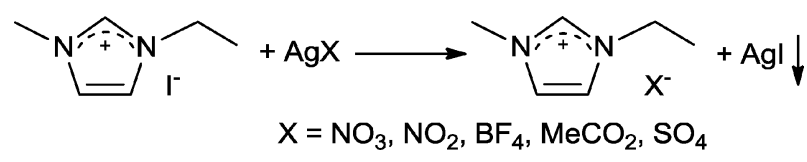

Scheme 1 The salt metathesis route used to prepare air and moisture stable ILs. 


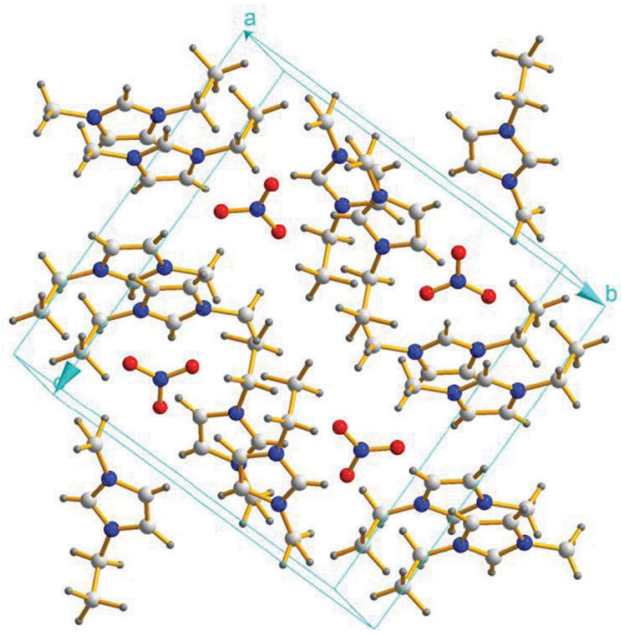

Fig. 1 The crystal structure of $\left[\mathrm{Emim}_{\mathrm{N}} \mathrm{NO}_{3}\right.$ reported in the original communication.

liquid at room temperature, and consequently some were studied using single X-ray diffraction analysis, which showed that certain anions interact strongly with water trapping it in the crystal (see Fig. 1).

Access to air stable ILs had a transforming effect on the field bringing diversity not only to IL structures but also potential applications. At that time the emphasis moved from finding applications in batteries to applications in catalysis. Combinations of stable cations and anions with a low viscosity and low vapor pressure were perceived to be ideal for industrial-scale synthesis replacing hazardous volatile organic solvents and shortly afterward some pioneering papers emerged that included further examples of air-stable ILs. ${ }^{8,9}$ Concurrently, the potential of air-stable ILs as electrolytes in dye-sensitized solar cells was explored by Grätzel et al. and today ILs are used in commercial cells. ${ }^{10}$ The in situ crystallization of room temperature ILs with low melting points and their structural analysis by X-ray analysis revealed the intricate network of hydrogen bonds present, ${ }^{11}$ interactions which are key to fully understanding the physical properties of ILs. These structural studies laid the foundation to what is a now a vast array of spectroscopic and theoretical studies concerning ILs.

While most research was focused on the physical properties of ILs Rogers and Davis et al. took another leap forward by considering their chemical properties, specifically, how to manipulate the structure of an IL to give specific chemical (and ultimately physical) properties by design. Although not their first paper on the topic, ${ }^{12}$ in 2001 Rogers and Davis et al. published a paper in Chem. Commun. that described modified ILs for the extraction of heavy metals, i.e. task specific ILs (TSILs). ${ }^{13}$ Their concept was to incorporate the extractant into the IL structure - appending urea-, thiourea- and thioethergroups onto imidazolium cations (Fig. 2) that were combined with the hydrophobic $\mathrm{PF}_{6}{ }^{-}$anion allowing $\mathrm{Hg}^{2+}$ and $\mathrm{Cd}^{2+}$ ions to be removed from aqueous solution.

Davis explained to us, 'First, I think it [the Chem. Commun.] put the concept of TSILs before a much larger audience than we had theretofore had - our earlier papers outlining the concept having appeared in more specialized journals. The second thing
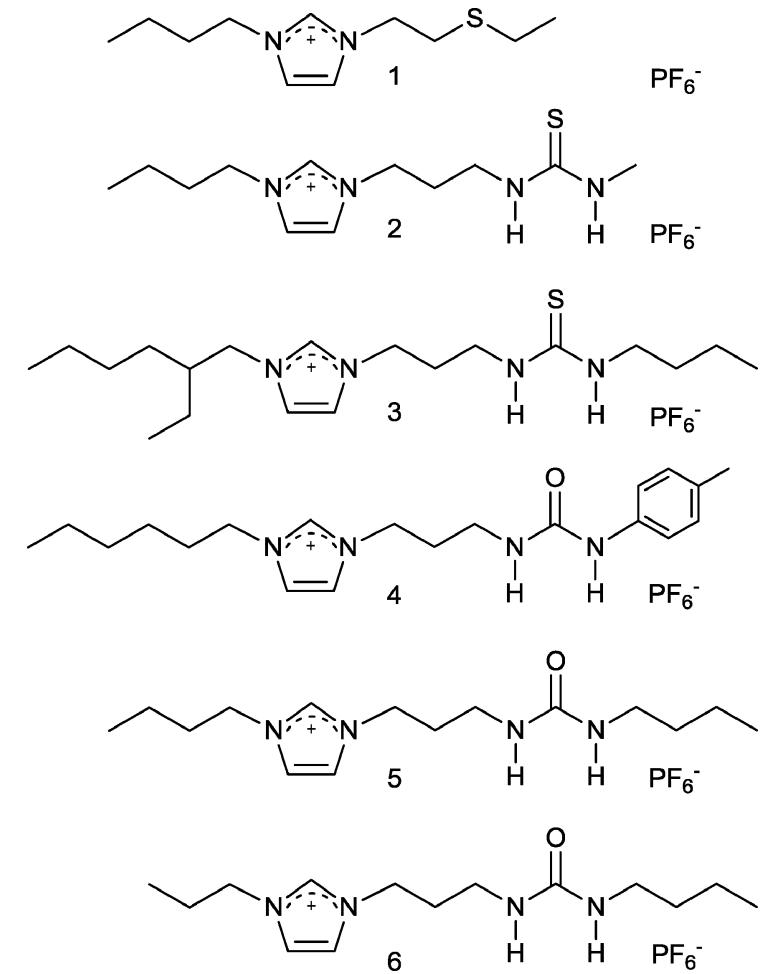

Fig. 2 TSILs designed for the extraction of toxic heavy metals.

was that the TSILs in this paper were designed by us for use in an application completely different than one we had worked with before.' Rogers concurred with the following statement, 'I think this paper added to the idea that if you wanted to, you truly could design the IL to be multi-functional; not just the solvent, but literally serve any other function you decided you wanted it to fulfill.' We concur with these remarks. Their TSILs were not simply an expansion of ILs, nor were they a revolution either; the result is something analogous to an iPhone providing a multitude of applications and opportunities. While some prefer to call TSILs functionalized ILs, the term was important (it first appeared in ref. 12e) as it helped to convince others that the notion of designer solvents was not a vague idea, but something tangible, achievable. And not surprisingly ILs entered a new era with ever increasing diversity and applications.

Today ILs are found in virtually every area of science and technology, ${ }^{14}$ and the reason for the now ubiquitous nature of ILs can be credited to a good extent to the two communications highlighted here. It is now known that some of the air-stable ionic liquids reported are not entirely air-stable and that TSILs can have more than one task. Nevertheless, Wilkes and Zaworotko laid the foundations for the explosive growth in IL chemistry and Rogers and Davis et al. expanded the field beyond traditional boundaries. Whatever the application it appears that ILs could be useful, from processing biomass ${ }^{15}$ to capturing carbon dioxide, ${ }^{16}$ from applications in mines to applications in space. Many of these applications depend critically on the physical properties of IL, which is in turn based on the nature of the ions, but with time the chemical properties of IL are becoming of greater value, which in turn is based on the functional groups attached to the ions. Perhaps it 
will not be too long before it is possible to dial up an IL for an application in the way we use an iPhone, perhaps iLiquids are already here... switchable liquids certainly are. ${ }^{17}$

\section{Acknowledgements}

We thank James H. Davis and Robin D. Rogers for interesting discussions. Sweet Home Alabama.

\section{References}

1 J. S. Wilkes, Green Chem., 2002, 4, 73.

2 P. Walden, Bull. Acad. Imp. Sci. St.-Petersbourg, 1914, 405.

3 H. C. Brown and H. W. Pearsall, J. Am. Chem. Soc., 1952, 74, 191.

4 H. L. Chum, V. R. Koch, L. L. Miller and R. A. Osteryoung, J. Am. Chem. Soc., 1975, 97, 3264.

5 J. L. Atwood, Advances in Chemistry Series, Inorganic Compounds with Unusual Properties, 1976, vol. 150, p. 112.

6 J. S. Wilkes, J. A. Levisky, R. A. Wilson and C. L. Hussey, Inorg. Chem., 1982, 21, 1263.

7 J. S. Wilkes and M. J. Zaworotko, J. Chem. Soc., Chem. Commun., 1992, 965.

8 P. A. Z. Suarez, J. E. L. Dullius, S. Einloft, R. F. de Souza and J. Dupont, Polyhedron, 1996, 15, 1217.
9 Y. Chauvin, L. Mussmann and H. Olivier, Angew. Chem., Int. Ed. Engl., 1995, 34, 2698.

10 P. Bonhôte, A.-P. Dias, N. Papageorgiou, K. Kalyanasundaram and M. Grätzel, Inorg. Chem., 1996, 35, 1168.

11 A. R. Choudhury, N. Winterton, A. Steiner, A. I. Cooper and K. A. Johnson, J. Am. Chem. Soc., 2005, 127, 16792.

12 (a) J. G. Huddleston, H. D. Willauer, R. P. Swatloski, A. E. Visser and R. D. Rogers, Chem. Commun., 1998, 1765; (b) J. H. Davis, Jr., K. J. Forrester and T. Merrigan, Tetrahedron Lett., 1998, 39, 8955; (c) J. H. Davis, Jr. and K. J. Forrester, Tetrahedron Lett., 1999, 40, 1621; (d) T. L. Merrigan, E. D. Bates, S. C. Dorman and J. H. Davis, Jr., Chem. Commun., 2000, 2051; (e) A. Wierzbicki, J. H. Davis, Jr., Paper No.14F, Proceedings of the Symposium on Advances in Solvent Selection and Substitution for Extraction, March 5-9, 2000, Atlanta, Georgia. AIChE, New York, 2000.

13 A. E. Visser, R. P. Swatloski, W. M. Reichert, R. Mayton, S. Sheff, A. Wierzbicki, J. H. Davis, Jr. and R. D. Rogers, Chem. Commun., 2001, 135.

14 N. V. Plechkova and K. R. Seddon, Chem. Soc. Rev., 2008, 37, 123.

15 R. P. Swatloski, S. K. Spear, J. D. Holbrey and R. D. Rogers, J. Am. Chem. Soc., 2002, 124, 4974.

16 E. D. Bates, R. D. Mayton, I. Ntai and J. H. Davis, Jr., J. Am. Chem. Soc., 2002, 124, 926.

17 P. G. Jessop, D. J. Heldebrant, X. Li, C. A. Eckert and C. L. Liotta, Nature, 2005, 436, 1102. 